\title{
Silicon $\Sigma 13(501)$ grain boundary interface structure determined by bicrystal Bragg rod x-ray scattering
}

\author{
P. B. Howes ${ }^{\mathrm{a}}$, S Rhead ${ }^{\mathrm{a}}$, M. Roy ${ }^{\mathrm{a}}$, C. L. Nicklin ${ }^{\mathrm{b}}$, J. L. Rawle ${ }^{\mathrm{b}}$, C. A. \\ Norris $^{\mathrm{b}}$ \\ ${ }^{a}$ University of Leicester, Department of Physics and Astronomy, LE1 7RH, England \\ ${ }^{b}$ Diamond Light Source Ltd., Diamond House, Harwell Science and Innovation Campus, \\ Didcot, Oxfordshire, OX11 ODE, England
}

\begin{abstract}
The atomic structure of the silicon $\Sigma 13(501)$ symmetric tilt grain boundary interface has been determined using Bragg rod x-ray scattering. In contrast to conventional structural studies of grain boundary structure using transmission electron microscopy this approach allows the non-destructive measurement of macroscopic samples. The interface was found to have a single structure that is fully four-fold coordinated. X-ray diffraction data were measured at Beamline I07 at the Diamond Light Source.
\end{abstract}

\section{Introduction}

Grain boundaries (GBs) in semiconductors have been studied for many years and remain of interest due to the importance of polycrystalline semiconductors in the microelectronics industry, interest in micromechanical materials, solar energy applications, and nanocrystalline Si $[1,2,3,4,5]$. The GBs can provide preferential sites for dopants [3, 6, 7], and act as low energy diffusion pathways. For low-energy tilt boundaries, which usually retain the fourfold coordination of the bulk $[1,3]$, the dominant electronic effects are assumed to be due to dopant segregation to the GB; however, intrinsic gap states localised to the GB may occur if the bonding is sufficiently distorted $[2,3,8]$. Understanding the atomic structure of semiconductor GBs is Rhead)

Email addresses: Paul.Howes@le.ac.uk (P. B. Howes), S.Rhead@warwick.ac.uk (S 
therefore highly desirable, however, their structural complexity makes both experimental and theoretical studies difficult.

There are several experimental methods for characterising GB structure, the most common of which is transmission electron microscopy (TEM) $[9,5]$. The development of high resolution TEM (HRTEM) and aberration corrected instruments allow atomic resolution. To determine interface structure it is necessary to image the crystals on both sides of the interface and this limits the method to pure tilt grain boundaries in which columns of atoms in both crystals are parallel to the viewing direction. In such circumstances a two-dimensional projection of a three-dimensional crystal can be obtained. The projection is difficult to interpret directly and a comparison with simulated images is often necessary. The main limitation is that TEM is a two-dimensional technique [10] and the grain boundary is averaged over the sample thickness though this can occasionally be overcome by taking images in two or more projections [11]. Other limitations include structural changes because of electron beam radiation damage to the sample. As the electron beam is weakly penetrating the sample has to be thinned to the extent that it is effectively destroyed to access it. In common with all microscopies, TEM is essentially a local technique.

Another approach is to predict the structure of the grain boundary theoretically. Realistic GB atomic models contain large number of atoms, often too many for ab-initio calculations. Empirical potentials, such as Tersoff or Stillinger-Weber, are often employed but they do not always correctly predict the lowest energy structure. Theoretical studies are also complicated by the fact that, for a given tilt angle, several possible grain boundary structures can exist physically and are observed experimentally. This has been shown by Rouvière et al. [10] who observed several possible grain boundaries for various tilt angles in both $\mathrm{Si}$ and Ge bicrystals. Because of this, some have suggested that the energetics of the grain boundary is not the complete story in correctly predicting the grain boundary structure $[2,10]$.

In this paper we present an x-ray diffraction study of the silicon $\Sigma 13(501)$ GB interface using Bragg rod scattering to determine the atomic structure. Diffraction measures the global average structure and is thus complimentary to microscopic studies. We exploit the large penetration of x-rays into matter to study, non-destructively, a macroscopic sample in a transmission geometry. 


\section{Background}

\subsection{Bragg rod scattering from interfaces}

The two-dimensional nature of crystalline surfaces and interfaces leads to streaks of scattering in reciprocal space that are known as Bragg rods. Some Bragg rods arise from the truncation of the crystal at a plane (surface or interface) and are formed from the tails of Bragg peaks overlapping in reciprocal space in the direction normal to the surface [12]. These rods are known as crystal truncation rods (CTR) [13] and are widely used to determine the atomic arrangement at surfaces. They have, however, not often been been applied to buried interfaces and never before to a wellaligned bicrystal interface. Previous x-ray CTR studies of interfaces include crystalline-amorphous interfaces $[14,15]$ and a $\mathrm{SrTiO}_{3}$ symmetric tilt grain boundary $[16,17]$. In the latter case, the sample was not very well aligned and the Bragg rod tails did not merge to form a continuous rod and, in addition, the data were complicated by a thin film geometry that introduced scattering from the top surface. Bragg rod scattering was also used to study the $\Sigma 5$ twist GB in gold bicrystals formed by sintering two thin films [18] but in contrast to this study, their 'relrods', analogous to fractional order Bragg rods from a reconstructed surface, were not CTRs and did not contain Bragg peaks from the bulk crystals.

\subsection{Coincidence GBs}

GB geometry is defined by the misorientation (angle and direction) of two crystals and the orientation of the plane boundary that forms the interface. Coincidence GBs, also known as 'special angle' GBs, occur when certain lattice points from the two crystals overlap leading to lattice of shared lattice points known as a coincidence site lattice (CSL). It can be shown that, for a symmetric tilt GB with misorientation angle, $\theta$, about an [010] axis in a cubic material, a coincidence boundary is formed if $\tan ^{-1}(\theta / 2)$ is a rational number. The coincidence site index, $\Sigma$, also known as the twin index is the inverse of the fraction of lattice points that are shared between the two crystal lattices. Coincidence GBs often have low energy, stable structures and have received a great deal of theoretical and experimental study [3].

Bragg rod scattering from coincidence GBs is particularly interesting. Because of the alignment of crystal lattices, there is a corresponding alignment of reciprocal space lattices. This means that one in $\Sigma$ Bragg peaks from the bicrystal will be shared in common. Furthermore, Bragg rods arise that are 
due to the termination of two different crystals at the common interface plane and these bicrystal truncation rods (BCTR) contain Bragg peaks from both crystals. The flat interface leads to streaks of scattering at every Bragg peak and for a perfectly aligned CSL interface these streaks overlap to form a continuous BCTR that is the coherent sum of scattering from each semi-infinite crystal and from the relaxed interface region containing the dislocation cores.

\subsection{Si $\Sigma 13(501)$ interface structure and geometry}

Different possible Si $\Sigma 13$ GB structures in which all the atoms are fourfold coordinated have been discussed by Morris et al. [2] and are shown in Figure 1. Several of the structures have been observed experimentally $[19,20]$ though Rouvière et al. [5] conclude that this interface shows only the single structure labelled (a) which is also the structure predicted to have the lowest energy [2].

The construction of the CSL is described at length by Sagalowicz and Clark [21]. The $\Sigma 13$ CSL unit cell has dimensions of $a_{1}=\frac{1}{2} \sqrt{26} a_{0}, a_{2}=a_{0}$ and $a_{3}=\frac{1}{2} \sqrt{26} a_{0}$, where $a_{0}=5.43 \AA$ is the Si cubic lattice constant. This CSL in turn generates the two-dimensional interface unit cell. The repeat distance along the boundary is $\frac{1}{2} \sqrt{26} a_{0}$ with the factor of $\frac{1}{2}$ arising because of extra lattice points from the face centred cubic unit cell.

The boundary contains a number of dislocations, with a total Burgers vector of $\mathbf{b}=[100]$. The individual dislocations that occur are either $\mathbf{b}=$

$\frac{1}{2}[110]$ and $\mathbf{b}=\frac{1}{2}[1 \overline{1} 0]$ pairs of edge dislocations or $\mathbf{b}=\frac{1}{2}[101]$ and $\mathbf{b}=$ $\frac{1}{2}[10 \overline{1}] 45^{\circ}$ mixed dislocation pairs [22]. Structures (e) and (f) are grain boundaries made up solely of edge dislocations, and are characterised, in projection, by a set of five-fold and threefold rings sharing a vertex. The structural units from which all of the grain boundaries can be constructed are illustrated in Figure 2.

\subsection{Keating Energy Minimisation}

In order to calculate the x-ray scattering from the bicrystal interface it is necessary to know the coordinates of the atoms that make up the relaxed dislocation cores as well as the strain the the top and bottom bulk silicon lattices. Following earlier x-ray diffraction studies of surfaces [24, 25] and interfaces [26] we used the classical Keating energy to relax the structure and ensure that it is physically reasonable. Though the Keating potential would not be expected to predict the exact details at the highly strained 


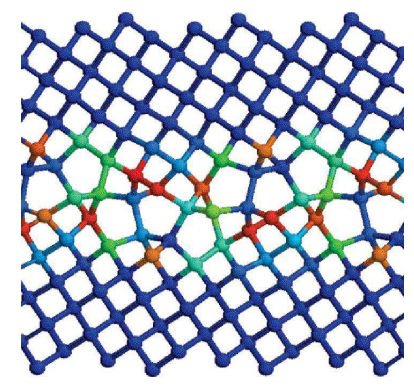

(a)

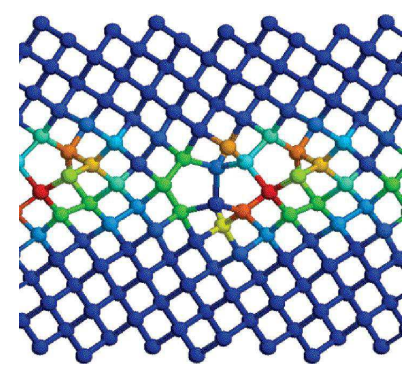

(d)

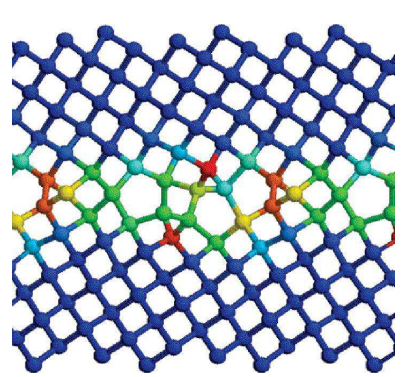

(b)

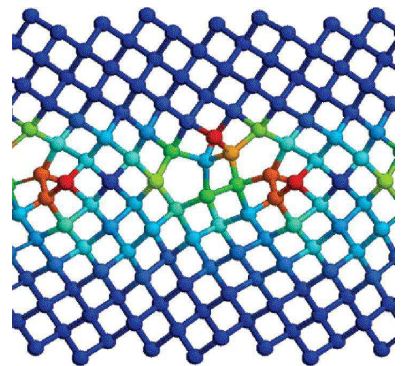

(e)

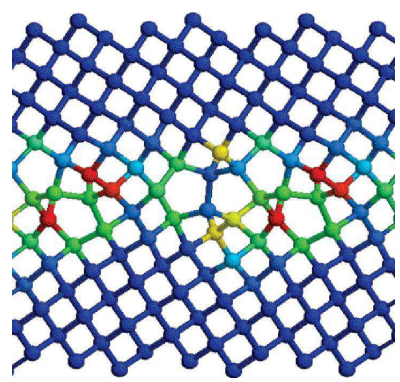

(c)

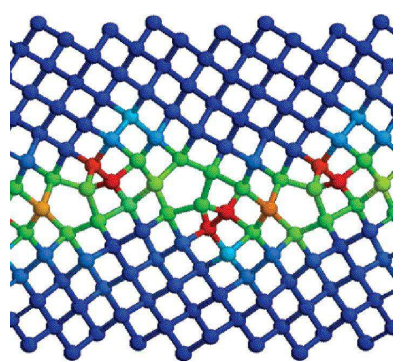

(f)

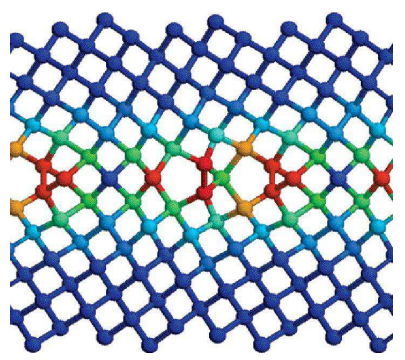

(h)

Figure 1: The possible $\Sigma 13$ interface structures proposed by Morris [2]. Starting with each of their models we used the Keating potential to relax the atomic positions to find the minimum energy structure. The atoms are colour coded to indicate each atoms contribution to the Keating energy with high energy atoms appearing hotter. Structure (g) has been excluded as it modelled incorrectly with the Keating minimisation. These structures are all fully four-coordinated. Note that the triangular and pentagonal loops spiral into the page and only appear to be wrongly coordinated in projection. 

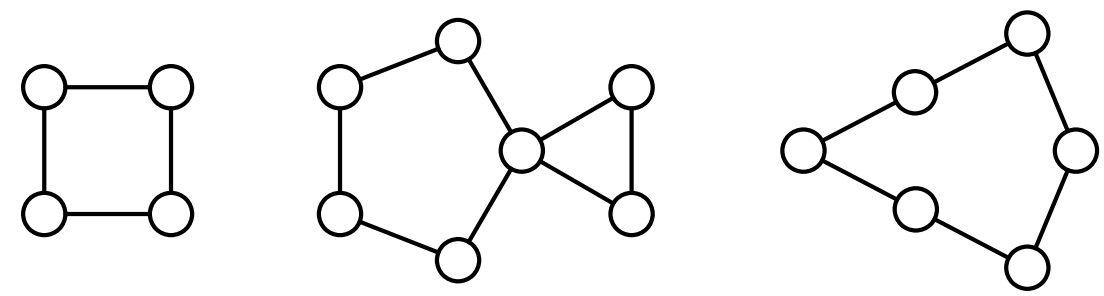

Figure 2: The structural units that make up the grain boundary structures. From left to right: a perfect crystal (unit A), pure edge dislocation (unit B) and a mixed dislocation (unit C). After [23].

dislocation cores, it gives accurate results for fully coordinated, bulk-like semiconductors and this describes the majority of the atoms contributing to the x-ray scattering. Significant contributions to the BCTR come from rigid body translations of the top crystal with respect to the bottom and from the strain field due to dislocations, both of which are accurately modelled by the Keating potential.

We used each of Morris' models in turn to determine the atomic configuration and bonding and then relaxed the structure to minimise the Keating energy. The models were constructed by first calculating the coordinates of two, overlapping, rotated bulk Si crystals. A boundary plane was inserted in such a way that only atoms belonging to the top crystal are on one side of it and atoms from the bottom crystal the other side giving the unrelaxed structure of the boundary. Finally an interfacial expansion (typically $2-7 \AA$ ) was introduced between the crystals to accommodate the grain boundary with its dislocation cores. Each Si crystal contained four $\Sigma 13$ unit cells (two horizontal and two vertical) meaning the interface structure repeated itself twice along the grain boundary. The atomic coordinates and bonds of the interface atoms were entered manually to ensure correct coordination of the unrelaxed bicrystal structure. Periodic boundary conditions were applied to the structure in the $x$ and $y$ directions creating an array of the Si $\Sigma 13$ bicrystal. The unrelaxed structures then underwent the Keating energy minimisation. The structures were relaxed after approximately a few thousand iterations.

The relaxed structures are illustrated in Figure 1 and the Keating energy was found to be similar across all of the structures, with the lowest energy structure having an energy approximately 10\% smaller than the highest energy structure (Table 1). The figures are colour-coded on a temperature scale 


\begin{tabular}{c|c|c|c}
\hline Structure & Bending & Stretching & Keating \\
\hline $\mathrm{a}$ & 577.82 & 18.97 & 596.79 \\
$\mathrm{~b}$ & 529.09 & 17.63 & 546.72 \\
$\mathrm{c}$ & 582.03 & 21.19 & 603.21 \\
$\mathrm{~d}$ & 568.66 & 24.37 & 593.02 \\
$\mathrm{e}$ & 526.29 & 28.39 & 554.68 \\
$\mathrm{f}$ & 570.41 & 30.71 & 601.12 \\
$\mathrm{~h}$ & 549.04 & 27.65 & 576.69 \\
\hline
\end{tabular}

Table 1: The Keating energies of the grain boundaries, in units of $\mathrm{mJ} . \mathrm{m}^{-} 2$. The bending and stretching components are shown to illustrate the relative weighting of each to the Keating energy.

illustrating each atom's individual contribution to the Keating energy. The hot (red) atoms have the most strain and the cool (blue) atoms have the least. The temperature scale is not absolute and varies from structure to structure. The strain is, as expected, highly localised at the interface. The structures are all fully four-bonded and are physically realistic.

A comparison of our Keating energy and the grain boundary energies obtained by Morris et al. is shown in Figure 3. The Keating energy depends only weakly on the structure of the grain boundary when compared to the tight-binding (TB) and ab-initio energies which show considerable differences. The TB and ab-initio simulations found structure (a) to be the lowest energy grain boundary and structure (h) to be the highest energy structure. Interestingly, structure (a) has the most dislocation cores whereas the grain boundaries that are structurally similar to the bulk, with a minimal number of dislocation cores, are all high energy. This is clearly not the case with the Keating model, which has structure (b) as the lowest energy and structure (c) as the highest energy. There is a lack of correlation between the ab-initio and classical potentials. This suggests that the dislocation cores are probably not modelled very well with the classical potential due to excessive bond bending and stretching [5]. However, we emphasise that the BCTR scattering is dominated by longer distance strain and rigid body translation of the crystals and that the Keating model is good enough to ensure that the model is physically realistic before the $\mathrm{x}$-ray scattering is calculated. 


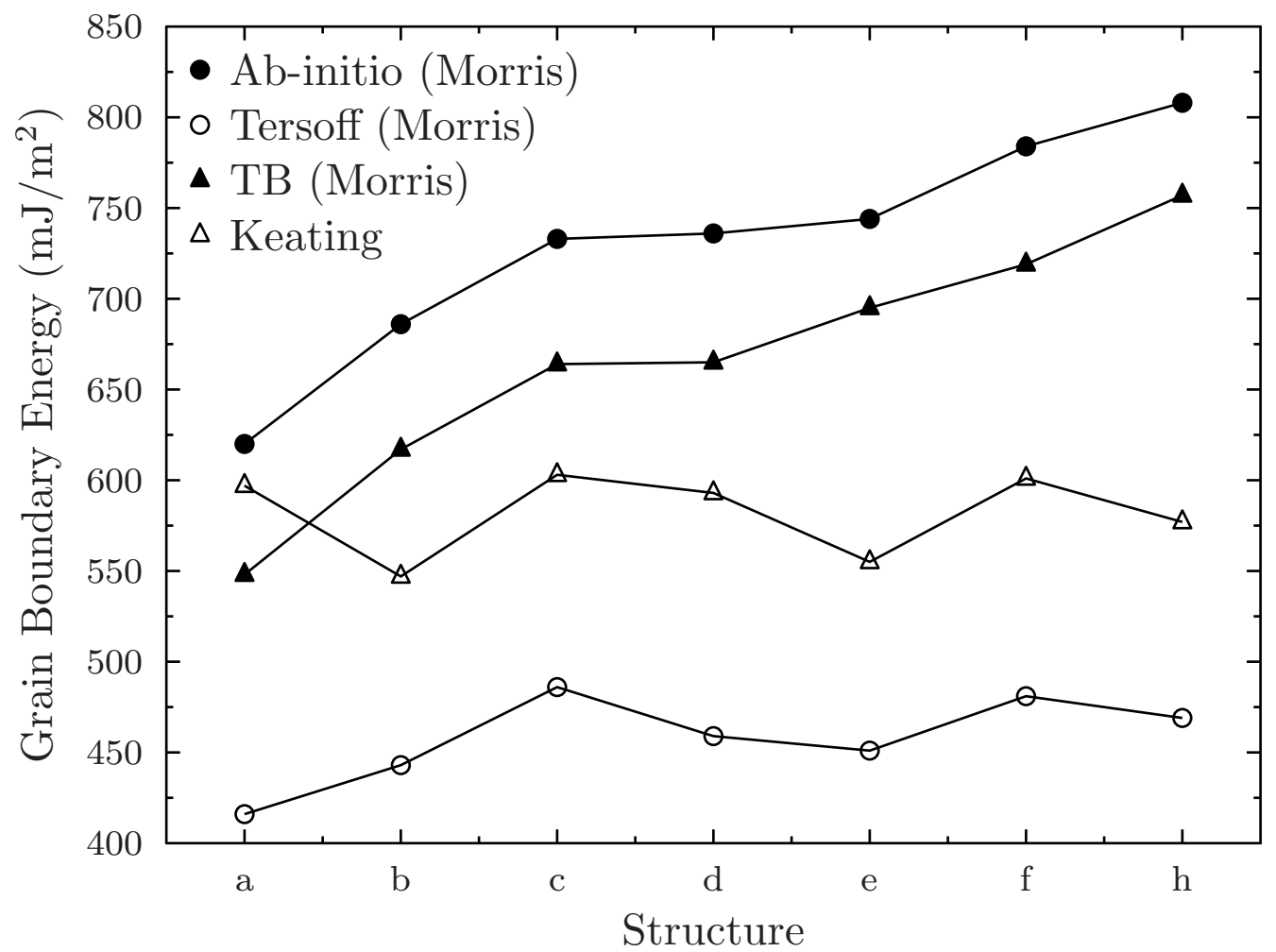

Figure 3: Grain boundary energies for the different structures identified in figure 1 . The Tersoff, tight binding and ab-initio energies are taken from [2]. 


\subsection{Simulated BCTR x-ray scattering}

We calculated x-ray scattering from each of Keating-relaxed models. As the $\sqrt{26} a_{0}$ unit cell was used in the Keating minimisation it was used, for convenience, as the unit cell for simulating the scattering. The relationship between the $\sqrt{26}$ Miller indices $(H, K, L)$ and the conventional cubic ones are given by the following transformations for the top crystal:

$$
\begin{gathered}
\left(\begin{array}{c}
H \\
K \\
L
\end{array}\right)=\left(\begin{array}{rrr}
1 & 0 & -5 \\
0 & 1 & 0 \\
5 & 0 & 1
\end{array}\right)\left(\begin{array}{c}
h \\
k \\
l
\end{array}\right) \\
\left(\begin{array}{c}
h \\
k \\
l
\end{array}\right)=\frac{1}{26}\left(\begin{array}{rrr}
1 & 0 & 5 \\
0 & 26 & 0 \\
-5 & 0 & 1
\end{array}\right)\left(\begin{array}{c}
H \\
K \\
L
\end{array}\right)
\end{gathered}
$$

with a similar transformation for the bottom crystal. In the following discussion, BCTRs will be indexed in terms of this cell and individual Bragg peaks in the rod will also be labelled using their conventional cubic indices for clarity.

The simulated BCTRs are calculated by summing the scattering from the top, bottom and interface regions in the kinematical approximation:

$$
f_{\text {total }}=f_{\text {top }} e^{i \mathbf{q} \cdot \mathbf{d}}+f_{\text {bottom }}+f_{\text {interface }}
$$

where $f_{\text {total }}$ is the total scattering, $f_{\text {top }}$ the scattering from the top crystal, $f_{\text {bottom }}$ the scattering from the bottom crystal, $f_{\text {interface }}$ the scattering from the grain boundary interface, $\mathbf{d}$ the interface expansion between the two crystals and $\mathbf{q}$ the momentum transfer. The interface expansion between the two crystals introduces a phase difference between the scattering from the top and bottom crystal. The scattering from the top and bottom bulk crystals is given by

$$
\begin{aligned}
f_{\text {bottom }} & =\frac{F_{\text {bottom }}}{2}\left(1-\frac{i \cos (\pi l)}{\sin (\pi l)}\right) \\
f_{\text {top }} & =\frac{F_{\text {top }}}{2}\left(1+\frac{i \cos (\pi l)}{\sin (\pi l)}\right)
\end{aligned}
$$

where $F_{\text {bottom }}$ is the structure factor of the CSL unit cell for the bottom crystal, $F_{\text {top }}$ is the structure factor of the unit cell for the top crystal (related to 
that of the bottom crystal by symmetry). The bracketed regions in Equation 4 is the CTR scattering from a semi-infinite crystal extending from $-\infty$ to 0 and truncated at a flat surface. The similar term in Equation 5 is the equivalent term for a semi-infinite crystal extending from 0 to $\infty$.

For each of the possible interface structures in Figure 1 there is an equivalent, mirror image structure with the same energy (reflected in the $K=0$ plane). This is because the interface has a lower symmetry than the single silicon crystal. Because the symmetry related structures have identical energies they are equally likely to occur in the macroscopic sample and we found that it was necessary to average $(H, K, L)$ and $(H, \bar{K}, L)$ to get agreement with the data.

\section{Experimental}

The silicon bicrystal used in this experimental study was grown by the Czochralski method [27] using two, carefully aligned, seed crystals [28] and was the subject of an early TEM study [21]. For the Si $\Sigma 13$ bicrystal, the [010] axes of the seeds are parallel and used as the growth direction. The crystals are misoriented by $2 \tan (1 / 5)=22.6^{\circ}$ about the $[010]$ axis and the boundary plane is $\{501\}$ in both crystals. The sample was a cylindrical rod of diameter $1.8 \mathrm{~mm}$ cut from the boule using a diamond core drill with the interface normal along the cylinder axis.

The sample was mounted on a (2+3)-type diffractometer [29] at the I07 beamline of the Diamond Light Source. A wavelength $\lambda=0.7276 \AA$ was chosen because the $\mathrm{x}$-ray beam penetrates the sample at this high energy but the quantum efficiency of the PILATS 100K (Dectris) area detector is also high enough. The transmission geometry ensured that the X-ray beam illuminated both crystals and the grain boundary interface simultaneously and the resulting BCTRs contained contributions from all three components. This geometry avoided the complications inherent in earlier thin-film experiments $[16,17]$.

Because the lattices of the upper and lower crystal are rotated $\pm 11.3^{\circ}$ with respect to the [001] direction the reciprocal lattices and the Bragg peaks of the two crystals are easily distinguishable. This is shown in Figure 4 for the $K=0$ plane. In order to align the interface with the beam, the coordinate system was set to that of the top crystal and the goniometer rotated to the calculated angles for the (080) Bragg peak. To locate the interface plane, the sample was moved along its axis perpendicular to the x-ray beam until 
the Bragg peak disappeared. A similar exercise using the (080) Bragg peak from the bottom crystal ensures that both crystals and the interface itself are illuminated.

After crystallographic alignment, BCTR rods were measured by first moving to a Bragg peak in either crystal and then moving in the $\pm L$ direction. Some BCTRs were measured with rocking curves using a point detector with fixed angular resolution (scintillation detector) and others using the stationary mode with the area detector for which the large acceptance angle of the detector collects the entire in-plane component of the rod for a given $L$. The integrated intensity is obtained using the box subtraction method presented in [30] for the area detector or conventional peak integration for the rocking scans. Structure factors were derived from the integrated intensities by application of geometrical correction factors [31, 29]. In addition, a correction factor that accounts for attenuation of the beam through the sample was calculated by numerical integration. The following eight rods were measured; $(\overline{8}, 0, L),(\overline{16}, 0, L),(\overline{4}, 1, L),(16, \overline{1}, L),(22,2, L),(18,3, L),(32,4, L)$ and $(18,5, L)$. The $(\overline{8}, 0, L),(\overline{16}, 0, L),(\overline{4}, 1, L)$ and $(22,2, L)$ rods were measured using the stationary mode and the remainder by rocking scans.

\section{Results}

BCTR scattering was calculated for each of the Morris models and was fitted to the whole measured data set comprising eight Bragg Rods by varying only two scale factors, one for rocking scans and one for the area detector measurements. This was necessary due to the different methods used to measure the BCTRs. The $\chi^{2}$ goodness of fit parameters are listed in 2 . Immediately it is seen that structure (a) gives a good fit to the data with a $\chi^{2}$ of 1.43 whereas none of the remaining structures fit satisfactorily with $\chi^{2}$ of 4.98 or greater. A typical BCTR from structure (a) is shown in Figure 5. It is readily apparent that only structure (a) fits the data. The good fit to the data was obtained without any structural refinement and assuming a flat interface. In principle, roughness of the interface could be accounted for [13]. We would expect any deviations from a flat interface to lead to a reduction in the intesity of the rod in between the Bragg peaks. However, an excellent fit was obtained in this case without including the effects of roughness. 


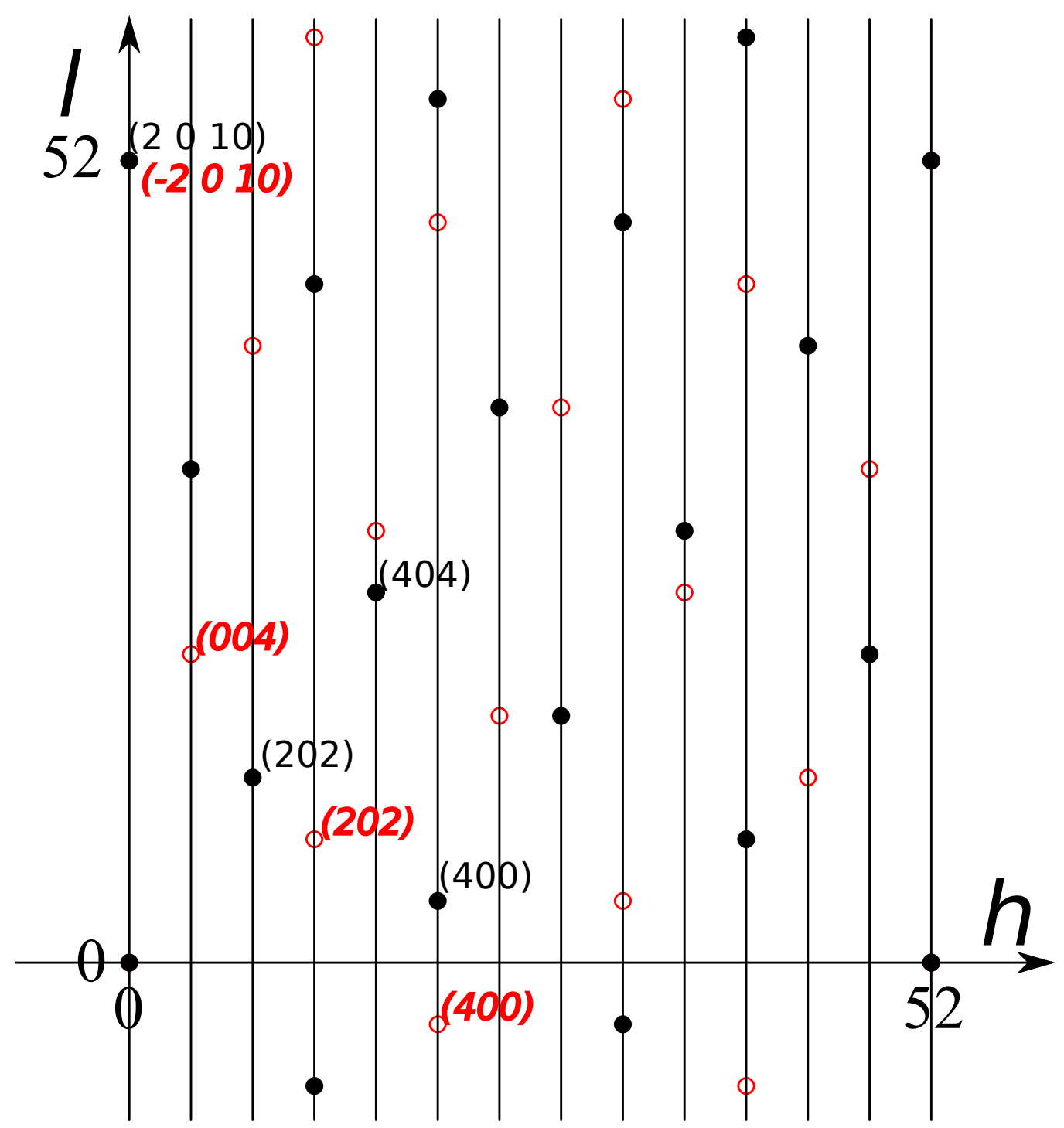

Figure 4: The reciprocal lattice of the bicrystal in the $K=0$ plane. The black circles belong to the reciprocal lattice of the top crystal and the red circles the reciprocal lattice of the bottom crystal. 


\begin{tabular}{c|c}
\hline Structure & $\chi^{2}$ \\
\hline (a) & 1.43 \\
(b) & 5.03 \\
(c) & 4.98 \\
(d) & 6.75 \\
(e) & 7.42 \\
(f) & 7.79 \\
(h) & 7.14 \\
\hline
\end{tabular}

Table 2: Agreement factors of the different grain boundary interface structures. The $\chi^{2}$ values are calculated using the whole data set comprising eight Bragg rods. Structure (a) is clearly favoured compared to the remaining structures.

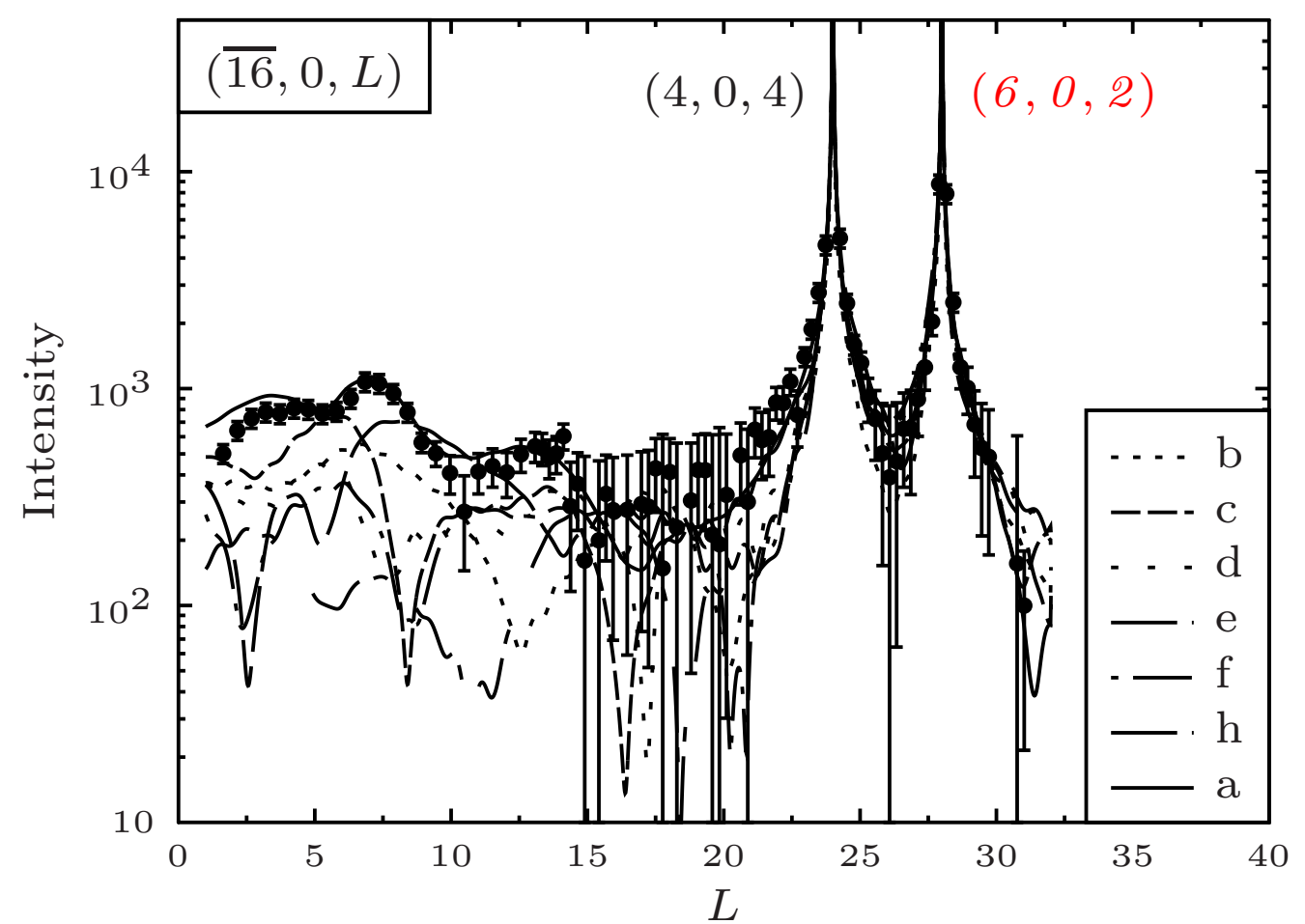

Figure 5: The $(\overline{16} 0 L)$ BCTR, one of eights such Bragg rods measured from the silicon $\Sigma 13$ grain boundary. The data (circles) are compared with the simulated scattering for each of the possible four-coordinated interface structures. Bragg peaks cell are labelled using conventional cubic coordinates for clarity; black from the top crystal and red (italic) from the bottom crystal. Each rod contains Bragg peaks from both crystals. Only structure (a) is a good fit to the data. 


\begin{tabular}{c|c}
\hline $2 \theta$ & $d(\AA)$ \\
\hline 11.88 & 3.52 \\
24.93 & 1.69 \\
23.39 & 1.79 \\
41.22 & 1.03 \\
36.23 & 1.17 \\
\hline
\end{tabular}

Table 3: : $2 \theta$ angles and $d$-spacings for the additional reflections due to precipitates in the interface plane.

\subsection{In-Plane Scans}

In-plane reflections were measured using the constant velocity mode [32] using the point detector. This approach is ideal for detecting sharp features in a wide angle scan. A wavelength of $0.7276 \AA$ was used and the peaks recorded at $L=0$. Unexpected, extra reflections were observed close to some BCTR peaks. Upon further observation using the area detector it became clear that these additional peaks were not due to Bragg rod scattering as they did not exhibit streaks characteristic of rod-like scattering. The sharpness of the peaks and their location reciprocal space indicate that they come from small crystallites oriented with the interface, most likely precipitates. The precipitates are located in the interface plane and were not observed if the sample was displaced so that the x-ray beam only illuminated one or other crystal.

Attempts were made to identify the precipitate material by considering the $d$-spacing listed in table 3 . The $d$-spacings were compared with standard powder diffraction tables. We were unable to fully determine the precipitate material as we have not fully mapped out reciprocal space but they are consistent with a theoretically obtained $\mathrm{SiO}$ diffraction pattern which is consistent with the precipitate material in $[19,21]$.

\section{Discussion}

Bicrystal truncation rods have been measured from a Si $513(501)$ symmetric tilt grain boundary. The precise misorientation between the two crystals leading to shared $\langle 501\rangle$ directions gives rise to a CSL in real space and many overlapping Bragg peaks in reciprocal space. The resulting Bragg rods are similar to CTRs commonly used to study surface reconstructions in that they contain contributions from both the semi-infinite bulk-like material and 
also the two-dimensional interface. Remarkably all of the BCTRs from this sample contain contributions from two bulk crystals. Thus, while the Bragg peaks in the rods belong to one crystal or the other, the rod itself is due to the truncation of both crystals. Our simulations show that the BCTR yield both information about the rigid body translation between the two crystals and the atomic structure of the grain boundary interface.

With careful optimisation of the experimental geometry and a suitable $\mathrm{x}$ ray energy, BCTRs are readily measurable at a third generation synchrotron such as the Diamond Light Source. Furthermore, these experiments do not require special or destructive sample preparation. The x-ray beam was able to pass through a macroscopic sample. By choosing a zero incidence angle and a narrow x-ray beam the interface was fully illuminated while background scattering from the bulk crystals was minimised.

Comparison between our experimental data and simulations gives a good fit to Morris' model (a) in agreement with their ab-initio and Tersoff simulations of GB energies. The good fit indicates that the Si $\Sigma 13$ interface is dominated by this single structure in agreement with the recent paper of Rouvière et al. [5] and in contrast to earlier studies that have observed both structure (a) and (d) have been observed [19, 20]. X-ray diffraction is insensitive to local defects and is an ideal technique for measuring the long range, ordered unit cell structure.

The sample used in this study was remarkably well aligned. The x-ray diffraction data showed no signs of peak broadening or splitting at all and the Bragg rods could be measured all the way from one Bragg peak to the next. This contrasts with earier studies of poorly aligned, sintered bicrystals $[16,17]$. The data in this study were fitted using a simple model with only a single structure and a flat interface. For other samples, where there may be a mixture of different structures existing in the same interface and the boundary may not be flat, additional analysis would be required [33].

Measurement of in-plane reflections unexpectedly showed extra peaks due to precipitates in the interface that are crystallographically aligned to the lattice. They may be silica in agreements with Sagalowicz and Clark who observed oxygen rich participates at the same interface [21] but further mapping of reciprocal space is required to definitively identify the material. 


\section{Conclusions}

We have a demonstrated a powerful new application of Bragg rod scattering to bicrystal interfaces. The BCTRs are formed from the coherent interference of CTRs from both sides of the interface along with scattering from the dislocation cores. Experiments performed at the Diamond Light Source have given experimental confirmation of the method and have been used to definitively distinguish between various possible interface structures at a Si $\Sigma 13 \mathrm{Si}$ (501) grain boundary interface.

BCTR scattering is a complimentary approach to the study of interfaces compared to local methods such as TEM. As a diffraction technique, it measure the global average unit cell atomic structure. It overcomes the limitations of TEM: Our technique is non-destructive as the large penetration of $\mathrm{X}$-rays into matter is exploited and the data is three-dimensional. In principle, BCTR studies are not limited to the study of tilt grain boundaries.

\section{Acknowledgements}

The authors would like to thank Professor Bob Pond of the University of Exeter for providing the boule from which the $\Sigma 13$ sample was cut and also for helpful discussions.

[1] Hornstra, J.. Models of grain boundaries in the diamond lattice i. tilt about [1 110$]$. Physica 1959;25:409.

[2] Morris, J.R., Lu, Z.Y., Ring, D.M., Xiang, J.B., Ho, K.M., Wang, C.Z., et al. First-principles determination of the $\Sigma 13\{510\}$ symmetric tilt boundary structure in silicon and germanium. Physical Review B 1998;58:11241-11245.

[3] Sutton, A., Balluffi, R.. Interfaces in Crystalline Materials. Oxford University Press; 1995.

[4] Grovenor, C.R.M.. Grain-boundaries in semiconductors. Journal of Physics C: Solid State Physics 1985;18:4079-4119.

[5] Rouvière, J.L., Lanon, F., Hardouin Duparc, O.. Atomic structures of Si and Ge $\Sigma=13$ [001] tilt grain boundaries studied by high-resolution electron microscopy and atomistic simulations. Philosophical Magazine 2013;93(10-12):1230-1249. 
[6] Maiti, A., Chisholm, M.F., Pennycook, S.J., Pantelides, S.T.. Dopant segregation at semiconductor grain boundaries through cooperative chemical rebonding. Physical Review Letters 1996;77:1306-1309.

[7] Bacmann, J.J., Papon, A.M., Petit, M., Silvestre, G.. Study of $\Sigma=5(130)$ symmetrical tilt boundary structure in germanium. Philosophical Magazine A: Physics of Condensed Matter Structure Defects 1985;51:697-713.

[8] Werner, J., Strunk, H., editors. Polycrystalline Semiconductors II; vol. 54. 1991.

[9] Bourret, A.. From the atomic structure of interfaces to their physical and chemical properties. Colloque de Physique 1990;51:C1-1.

[10] Rouviere, J.L., Bourret, A.. analysis of structures of symmetrical [001] tilt grain boundaries in silicon and germanium. Colloque de Physique 1990;51:329.

[11] Van Aert, S., Batenburg, K.J., Rossell, M.D., Erni, R., Van Tendeloo, G.. Three-dimensional atomic imaging of crystalline nanoparticles. Nature 2011;470(7334):374-377.

[12] Kaganer, V.M.. Crystal truncation rods in kinematical and dynamical x-ray diffraction theories. Phys Rev B 2007;75:245425.

[13] Robinson, I.K.. Crystal truncation rods and surface-roughness. Physical Review B 1986;33:3830-3836.

[14] Robinson, I., Waskiewicz, W., Tung, R., Bohr, J.. Ordering at $\mathrm{Si}(111) / \mathrm{a}-\mathrm{Si}$ and $\mathrm{Si}(111) / \mathrm{SiO}_{-}\{2\}$ interfaces. Physical Review Letters 1986;57(21):2714-2717.

[15] Renaud, G.. Oxide surfaces and metal/oxide interfaces studied by grazing incidence x-ray scattering. Surface Science Reports 1998;32(12):5 90 .

[16] Kazimirov, A., Zegenhagen, J., Denk, I., Maier, J., Smilgies, D.M., Feidenhans'l, R.. X-ray characterization of a $\mathrm{SrTiO}_{3}$ bicrystal interface. Surface Science 1996;352:875-878. 
[17] Feidenhans'l, R., Kazimirov, A., Smilgies, D.M., Jiang, Q., Zegenhagen, J.. X-ray scattering investigation of a $\mathrm{SrTiO}_{3}$ (103) bicrystal interface. Philosophical Magazine Letters 1998;78(1):51-57.

[18] Taylor, M.S., Majid, I., Bristowe, P.D., Balluffi, R.W.. Structures of [001] twist boundaries in gold. i. measurement and use of absolute boundary x-ray structure factors. Phys Rev B 1989;40:2772-2778.

[19] Kim, M., Carpenter, R., Chen, Y., Schwuttke, G.. Interaction between metal impurities and small-angle grain boundaries on recombination properties in multicrystalline silicon for solar cells. Ultramicroscopy 1992;40:258.

[20] Chisholm, M., Maiti, A., Pennycook, S., Pantelides, S.. Atomic configurations and energetics of arsenic impurities in a silicon grain boundary. Physical Review Letters 1998;81:132.

[21] Sagalowicz, L., Clark, W.. grain-boundary dislocations in a $\Sigma 13$ grainboundary in silicon .1. analysis of the possible grain-boundary structures and dislocations. Philosophical Magazine A 1995;72:545.

[22] Lazebnych, V.Y., Mysovsky, A.. Theoretical modeling of the structure of tilt grain boundaries in crystalline silicon. Physics of the Solid State 2012;54(12):2357-2361.

[23] Shenderova, O.A., Brenner, D.W., Yang, L.H.. Atomistic simulations of structures and mechanical properties of polycrystalline diamond: Symmetrical $\langle 001\rangle$ tilt grain boundaries. Physical Review B 1999;60:7043-7052.

[24] Pedersen, J.S.. Surface relaxation by the keating model: A comparison with ab-initio calculations aind x-ray diffraction experiments. Surface Science 1989;210(12):238 - 250.

[25] Pedersen, J.S., Feidenhans'l, R., Nielsen, M., Grey, F., Johnson, R.L.. X-ray diffraction study of the ge(111) $5 \times 5$-Sn and ge(111) $7 \times 7-\mathrm{Sn}$ surfaces. Physical Review B 1988;38:13210-13221.

[26] Rawle, J.L., Howes, P.B.. Modelling x-ray scattering from quantum dots using Keating energy-minimised structures. The European Physical Journal - Special Topics 2009;167:47-52. 
[27] Czochralski, J.. Ein neues verfahren zur messung der kristallisationsgeschwindigheit der metalle. Z Phys Chem 1918;92:219.

[28] Lu, Y.. Silicon bicrystal growth. Journal of Crystal Growth 1986;79:322.

[29] Vlieg, E.. A (2+3)-type surface diffractometer: Mergence of the zaxis and (2+2)-type geometries. Journal of Applied Crystallography 1998;31:198-203.

[30] Schlepütz, C.M., Herger, R., Willmott, P.R., Patterson, B.D., Bunk, O., Brönnimann, C., et al. Improved data acquisition in grazingincidence X-ray scattering experiments using a pixel detector. Acta Crystallographica Section A 2005;61(4):418-425.

[31] Vlieg, E.. Integrated intensities using a six-circle surface x-ray diffractometer. Journal of Applied Crystallography 1997;30:532-543.

[32] Silfhout, R.G.v.. X-ray diffractometer for structural studies of surfaces and interfaces. Journal of Applied Crystallography 2000;33(6):13421350 .

[33] Vlieg, E., Vanderveen, J.F., Gurman, S.J., Norris, C., Macdonald, J.E.. X-ray-diffraction from rough, relaxed and reconstructed surfaces. Surface Science 1989;210:301-321. 